\title{
XLIV. On the numerical relation between the index of refraction and the wave-length within a refractive medium and on the limit of refraction
}

\section{T. Pelham Dale M.A.}

To cite this article: T. Pelham Dale M.A. (1888) XLIV. On the numerical relation between the index of refraction and the wave-length within a refractive medium and on the limit of refraction, Philosophical Magazine Series 5, 25:156, 325-338, DOI: $10.1080 / 14786448808628198$

To link to this article: http://dx.doi.org/10.1080/14786448808628198

Published online: 29 Apr 2009.

Submit your article to this journal

Ш Article views: 2

View related articles $\sqsubset$ 
LONDON, EDINBURGH, AND DUBLIN

\section{PHILOSOPHICAL MAGAZINE}

AND

\section{JOURNAL OF SCIENCE.}

[FIFTH SERIES.]

$M A Y 1888$.

XLIV. On the Numerical Relation between the Index of Refraction and the Wave-length within a Refractive Medium, and on the Limit of Refraction. By T. Pelham Dalf, M.A.*

THE following equation (see Sir Geo. Airey's Treatise on 1 the Undulatory Theory, ed. 1877, p. 93) is proposed as expressing the relation between wave-length and velocity of propagation in an isotropic medium :-

$$
v=\sqrt{\left(1-\frac{1}{2^{\frac{3}{2}}}\right) \frac{m}{h}} \cdot \frac{\sin \frac{\pi h}{\lambda}}{\frac{\pi h}{\lambda}} . . . . .
$$

$v$ is here the velocity of propagation, $h$ the distance between the undulating particles, $\lambda$ the wave-length, $m$ the absolute force of attraction, supposed to follow the law of the inverse square.

For our purpose this may be put under the form

$$
v=k \sin \frac{\pi h}{l} / \frac{\pi h}{l} \ldots . . \cdot . \cdot . \cdot .
$$

It is convenient to reserve $\lambda$ to si gnify the wave-length in free æther, and to use $l$ for the wave-length within the medium. The values of the wave-lengths in free æther are those given in the text-book of Glazebrook and the tables of Lupton.

Now, knowing the index of refraction for any fixed line by observation of the index $\mu$, the corresponding value of $l$ is given by the equation

$$
l=\frac{\lambda}{\mu}
$$

* Communicated by the Physical Society : read February 11, 1888.

Phil. Mag. S. 5. Vol. 25. No. 156. May 1888. 
326 Mr. T. P. Dale on the Index of Refraction and

A convenient notation is $l_{A}, \lambda_{A}, \mu_{A}, \& c$., for index and wave-lengths of the line A \&c.

Now if $\mathrm{V}$ be the velocity of propagation in free æether,

whence from $(\beta)$ we have

$$
v=\frac{\mathrm{V}}{\mu}
$$

$$
\frac{\pi h v}{l}=k \sin \frac{\pi h}{l} .
$$

Calling $\frac{\pi h}{l}, \theta$, we have

$$
\frac{\pi h v}{l}=k \sin \theta
$$

for any other value $l_{1}$ corresponding to index $\mu_{1}$ we have

and

$$
\frac{\pi h v}{l_{1}}=k \sin \theta_{1}
$$

or

$$
\frac{\pi h}{l_{1}}=\theta_{1}
$$

$$
\theta_{1}=\frac{l}{l_{1}} \theta . . . \quad . \quad . \quad . \quad .
$$

Hence if we can find a value of $\theta$ for any one wave-length and index, all the others can be found from the observed wave-lengths and corresponding indices.

The angle $\theta$ can be readily found, if we know two indices, one corresponding to any given wave-length, and the other corresponding to double the wave-length, both within the medium.

For

$$
\frac{\pi h}{l}=\theta
$$

then

$$
\begin{aligned}
\frac{\pi h}{2 l} & =\frac{\theta}{2}, \\
\frac{\pi h v}{l} & =k \sin \theta=2 k \sin \frac{\theta}{2} \cos \frac{\theta}{2}, \\
\frac{2 \pi h v^{\prime}}{2 l^{\prime}} & =2 k \sin \frac{\theta}{2} ; \\
\ddots \frac{v}{v^{\prime}} & =\cos \frac{\theta}{2},
\end{aligned}
$$

or

$$
\frac{\mu_{2 l}}{\mu_{l}}=\cos \frac{\theta}{2} \cdot \text {. . . . . . . . }
$$


Now $\mu$ and $\mu_{1}$ can be found either by observation or interpolation. If $\mathrm{H}$ be the line chosen, then, practically, the index of a wave-length twice as long in the medium will not be far from $A$; this we may call $\mu_{2 H}$ and speak of it as the index of the octave below.

It is evident that if $k$ remain constant, $\frac{k \sin \theta}{\theta}$ continually approaches $k$ as $\theta$ diminishes. Now if $l$ be increased and $h$ and $m$ remain constant, $\theta$ diminishes ; hence there must be a limit of refraction in the case of waves of very great length. This limit, denoted by $\nu$, is evidently found by the equation

$$
\nu=\frac{\mu \sin \theta}{\theta} \text {. . . . . . . . . }
$$

This is in effect the limit found by Cauchy in a somewhat modified form.

Since

$$
\begin{aligned}
v & =\mu \frac{\sin \theta}{\theta}, \\
& =\mu_{1} \frac{\sin \theta^{\prime}}{\theta^{\prime}},
\end{aligned}
$$

it follows on substitution of $\frac{\pi h}{l}$ for $\theta$ and $\frac{\lambda}{\mu}$ for $l$, and dividing out, that

$$
\sin \theta=\frac{\lambda}{\lambda_{1}} \sin \theta_{1} \ldots . . \quad . \quad . \quad .
$$

Combining this with the equation above,

$$
\theta=\frac{l_{1}}{l} \theta_{1}, \quad . \quad . \quad . \quad . \quad . \quad . \quad . \quad .
$$

we can find a value of $\theta$ which satisfies both these equations from any two values of wave-length and corresponding index. And from this value of $\theta$ the corresponding values of $\theta$ to any other index and wave-length can be found.

In practice this equation can be solved by trial without much trouble, especially when a large number of indices of substances of similar refractive power have to be investigated, when a table may be made once for all, and from this $\theta$ may be found by inspection.

The wave-lengths made use of in this paper are generally those corresponding to lines $\mathrm{A}$ and $\mathrm{H}$. These are both within the visible spectrum, yet far enough apart to render errors of observation of little consequence. In the case of the liquids examined I am altogether indebted to Dr. Gladstone, who furnished me with those contained in Table III. for the purposes $\mathrm{Z} 2$ 
of this investigation. When the line $\mathrm{B}$ is given, the octave ray to $\mathrm{H}$ may be obtained from $\mathrm{A}$ by interpolation, and these values were calculated by this method. It gives results sufficiently near the truth, but has been verified in case of $\mathrm{CS}_{2}$ (temp. $\left.1^{\circ} .5\right)$ and mint hydrocarbon by reference to the two indices $A$ and $H$, so that the table depends on these two indices only. It will be seen that in the cases examined $\frac{v-1}{d}$ is constant, within the limits of observation, unaffected by temperature.

Dr. Gladstone and myself, in our joint paper, read before the Royal Society, May 6, 1863, and printed, p. 31.7, in the Transactions, have pointed ont that in consequence of an accidental relation amongst the coefficients used in Cauchy's series, that the equation

$$
\nu=\mu_{\mathrm{H}}-3\left(\mu_{\mathrm{F}}-\mu_{\mathrm{B}}\right)
$$

holds with considerable accuracy in the cases examined. It appears generally to give a fair approximation to the limit*, and thus to interpolate indices within the visible spectrum.

While, however, this investigation was in progress Prof. Langley's first paper on the determination of wave-lengths in the invisible spectrum appeared (Phil. Mag. March 1884). Ho there gives the wave-lengths of lower heat-rays down to length $2 \cdot 030$, and shows that the observed indices of refraction are not only below the limit obtained by Cauchy's formula, but even below those found by other formulæ. The material of his prism was flint glass. I found that the values derived from $\theta_{\mathrm{H}}$ and $\theta_{2 \mathrm{H}}$, while fairly accurate in the visible spectrum, were increasingly discordant in the longer wave-lengths. If $\theta_{\mathbf{H}}$ were increased the lower indices were found more accordant; but then the visible spectrum was not adequately represented within the limits of any error which could be imagined possible. This seemed to throw a doubt on the existence of a limiting value. It appeared, however, that the glass prism absorbed the longer waves considerably. The Phil. Mag. for May 1886 contained another paper by Prof. Langley, the material of the prism being rock-salt. On comparing the values found by observation with those furnished by the above equations, the agreement was sufficiently close to encourage investigation, and, accordingly, the calculations were carried further, the approximation being to single seconds in the value of $\theta$ and every value found.

The results are embodied in Tables I. and II. It will be

* The divergence in case of $\mathrm{CS}_{2}$ is, however, considerable, and $\nu$ is too large in comparison with that given by the above equations. Especially does this occur in highly dispersive media. 
seen in the case of rock-salt that the agreement between the values obtained from the sine-which involve only wavelengths in free wher-and those obtained from the arc, which involve the two indices, and which should be equal to the former, are very close. The errors, it will be observed, are of small amount and not of uniform sign, possibly, however, showing a small tendency to increase on the side of angle from the sine in the case of the lower indices. Turning to the table of indices in the case of flint glass, we see that the angle for $\mathrm{H}, 24^{\circ} 18^{\prime} 40^{\prime \prime}$, gives fairly accordant values down to wavelength 0.940 , and not firr from the truth to $1 \cdot 270$; after this the values increase in the case of the sine over that of the arc, or, in other words, the calculated index comes out too large. This is the more important because the proportionate increase on so small an angle as $4^{\circ}$ makes a large increase in the resulting index. If the value of $\theta_{\mathrm{H}}$ be increased, this difference is diminished in the lower values; but it would require an increase far greater than could possibly be allowed as due to errors of observation in order to include the lower values, and the upper would become greatly discordant with. observation.

It was, however, noticed by Prof. Langley that the flintglass prism appeared strongly absorbent of the lower rays. It would no doubt be unsafe to reason very confidently from results obtained from only two substances. We may remark, however, that the approach to limit of refraction, if it exist, must display itself in observation by a small increase in the index for a large proportionate increase in the wave-length. Any disturbing cause then must become, proportionately, also increasingly effective as the wave-length increases. Hence must arise a tendency to mask the limiting value if attempted to be found by observation. Now in the case of flint glass we can hardly suppose but that some effect is produced by the change of temperature which results from the passage of the heat-rays through the medium. In the case of fluids the heat lowers the index, and thus the limit would be lowered also, and in somewhat greater proportion. It is, in the absence of sufficient data, not easy to say what would be the effect of this change of temperature in solids. In the case of glass, the course of the ray through the medium would possibly be raised in temperature above the surrounding mass, and thus there would be a cylinder of glass through which the pencil of rays passed, which might be in a state of constraint as compared with that around it. That a state of constraint exists is shown by the glass "flying" when suddenly heated.

However this may be, it seemed desirable in the first place 
to continue the investigation with the data at hand, and to calculate the value of the limit as given by the above equations, using as data two observed indices only. The results are given in the tables, and will be seen to include a considerable number of fluids differing widely in specific gravity, optical properties, and chemical constitution. The list it will be noticed includes certain isomeric bodies and also several differing by a single component.

The first fluid examined was bisulphide of carbon at different temperatures, the data for which were furnished by Dr. Gladstone to me for the purpose of this investigation. It will be seen that the quantity $\frac{v-1}{d}$ is practically a constant. The same result is observable in the case of mint hydrocarbon and benzene. The range of temperature is not indeed very large, but within the limits taken it is evident that the specific gravity and the quantity $\nu-1$ increase or diminish pari passu, and there is but little doubt that this relationship obtains generally. It would be very desirable indeed to have longer ranges of temperature * if these could be procured without corresponding errors which high temperature would almost certainly have a tendency to introduce.

It is of interest evidently, in the case of a highly dispersive substance, to determine how far the different indices yield the same limit, as they clearly should do if the formulæ are exact. For the determination of this a value of $\theta$ was chosen which should yield the value which would satisfy $\theta_{\mathrm{A}}$; and then wavelengths of lines B CDE F and $G$ in free zther and in the medium were treated so as to find $\theta_{\mathrm{B}} \& \mathrm{c}$. If the sine and arc give the same result, then calculation and observation agree. It will be seen that in the case of $\mathrm{CS}_{2}$ there is an outstanding difference which amounts to not quite three minutes of arc, and increases from A to $H$. This is equivalent to saying that the formulæ give correct results to the third place in the case of $\mathrm{CS}_{2}$. This is not so good as the rock-salt in Table II. The index of $\mathrm{G}$ is most affected. The difference was noticed by the late Professor Baden Powell, in his ' Undulatory Theory as applied to the Dispersion of Light,' London, 1841. This matter deserves further investigation, but it is a matter also which will involve a considerable amount of calculation; thus it seemed right to postpone this for the present, as being better

* This is especially desirable near boiling and freezing points. It is possible that the specific gravity at boiling-point might reveal relations at present masked by the arbitrary character of specific gravities taken at any temperature convenient for observation. 
policy to obtain first a sufficient number of values of $\boldsymbol{\nu}$ in case of media varying considerably in optical properties.

It seemed also desirable to include in the choice of bodies several which were isomeric. There is a certain analogy in these to a body at different temperatures, as in either case we are dealing with the same chemical elements. The result is, as will be seen from examining the tables, that in many cases $\frac{v-1}{d}$ is very nearly a constant. In no case yet examined is the divergence very great. Thus in benzene and styrolene, cresol, metacresol, and benzil alcohol, benzil chloride and chlorotoluine, methyl citraconate and methyl mesaconate, the agreement is close; but in case of picoline and aniline, as also acetone-with which butyric ether agrees -as compared with allyl alcohol there is a difference of about three units in the second place*. It may be merely an accidental coincidence, but it appears that the quantity $\frac{\sqrt{ } \bar{h}}{d}$ in these two last-mentioned cases is nearly the same, but in others the divergence is found to be greater. This also requires further investigation.

It must, however, be remembered that in all these the limit is derived solely from $\mathrm{A}$ and $\mathrm{H}$. It is evident, then, that if either of these indices for $\mathrm{A}$ or $\mathrm{H}$ are in error, or are affected by anomalous dispersion, all the rest will be affected. Yet, considering the nature of the investigation,-which is, given certain indices to find the rest-it is evident that the fewer which are assumed as data the more confidence we may have, if we find that the rest are all calculable within reasonable limits. This, then, is the result of the present investigation : given two indices, all the rest can be found within limits, which are in a considerable number of instances very fair approximations to the truth. As, however, in the cases observed the outstanding differences are in the same direction, and increase apparently towards the more refrangible end of the spectrum, we have an indication of law, which may be sought for both in the circumstances of the experiment and in the mathematics of the problem.

It will be observed that the effect of substitution on the quantity $\nu-1$ is very marked indeed. This is shown in the case of ethyl sulphide and ethyl thiocarbamide, and still more in that of the substitution of iodine and bromine for chlorine. Iodine is peculiarly effective in increasing the

* But in these latter the specific gravity is evidently too high in the case of acetone, and in a less degree with butyric ether. 
specific gravity in far greater proportion than it raises the value of the refractive and dispersive powers. No simple relation between either density and $v-1$ or the quantity denoted by $h$ is as yet apparent. I have tried the square and cube, and the square root and cube root of $h$, as well as the simple power. I have noticed above that $\frac{\sqrt{h}}{d}$ is in case of some isomeric bodies nearly a constant, but this may be a mere coincidence. It is, however, worth noticing, as $\sqrt{h}$ enters the coefficient $k$ of Airey's equation cited above. When the logarithms are at hand, the calculations are so simple that even in the absence of any theoretical considerations it seemed-worth while to try them.

It will be observed that the equation, by $(\zeta)$ and $(\eta)$,

$$
a \sin \theta=\sin m \theta
$$

is impossible if $a \sin \theta$ is greater than unity. Now as $a$ is the ratio of two wave-lengths in free xther, denoted in this investigation by $\lambda: \lambda_{1}$, we may ask what happens if the ratio of $\lambda_{1}$ the upper wave-length to $\lambda$ the lower exceeds the ratio unity. Now in all cases yet examined, with the exception of selenium, this shorter wave-length is beyond violet and indeed the visible spectrum. In selenium it must lie somewhere near $\mathbf{E}$; in solid phosphorus and sulphur it is beyond the furthest limit of the spectrum towards its more refrangible end, given by Prof. Langley, i.e. 0.3727. The results are given for these three substances in 'Table IV. annexed. It will be seen that phosphorus reaches the limit at about 2684 and sulphur at nearly 2664 . We may observe as a coincidence that the behaviour of selenium in relation to light is remarkable, while its red colour shows that the violet end of the spectrum is absorbed. The results in Table V. are supplemental, calculated to the nearest minute, from determinations recently supplied by Dr. Gladstone, of $\nu$ for water and alcohol.

\section{Abstract of Results.}

1. That the relationship between the limit of refraction ( $v)$ found by the equation $a \sin \theta=\sin m \theta$, where $a$ is the ratio of two wave-lengths in free rether and $m$ the corresponding ratio in the medium, give a value which has the property expressed by the equation $\frac{\nu-1}{d}$ a constant, where $d$ is the density of the medium and $v$ its limit of refraction at the same temperature. 
2. That in isomeric bodies the same relation obtains closely in a large number of the bodies examined.

3. In all the cases examined $\nu$ is a quantity not very far below $\mu_{A}$.

4. That the substitution of one chemical element for another has a very great influence on the quantity $\frac{v-1}{d}$, and substances with high chemical equivalent have, as far as examined, a far greater influence in increasing the density than in raising the limit.

5 . That the equation $a \sin \theta=\sin m \theta$ gives in the cases examined an approximate value for all the other indices, two being assumed as data. In the case of rock-salt this holds good far down in the invisible heat-spectrum, and also in the ultraviolet, but fails somewhat at a certain point in the flint-glass spectrum, and also, to a less degree, at the upper end of the visible spectrum of bisulphide of carbon.

It is evident that for a certain value of $a$ and $\theta$ the equation $a \sin \theta=\sin m \theta$ becomes impossible. This would mean that there is an upper limit which is given by a certain value of wave-length, beyond which the formula gives impossible results. In the case of selenium only, of the substances examined, is this limit within the visible spectrum. The analogy with the critical angle of total reflexion gives this circumstance considerable interest.

It is evident, then, in the present state of our data, that in all cases examined the formula of Sir Geo. Airey gives an approximate value of an index by means of two others, and in this way they can be calculated; but that, as a rule, the lower indices come out too high as compared with the upper. A cause for this may perhaps be suggested in the heating of the medium due to the absorption of the rays; and as there seems to be evidence of absorption of short waves above the longer ones, given by recent stellar photometric determinations, this absorption would perhaps, if included in the conditions of the problem, account for some of the outstanding difference. This and anomalous dispersion -itself apparently depending on absorption-opens an interesting field of inquiry, and in this direction I propose to pursue the investigation.

Since the above was written I have been informed by Dr. Gladstone that he in some cases used a screen of solution of alum, but found that practically nothing was gained by its use. This fact, then, must be taken into account in any attempt to explain the outstanding differences between theory and observation. 
$334 \quad$ Mr. T. P. Dale on the Index of Refraction and

Table I.-Langley's Flint-glass Prism.

\begin{tabular}{|c|c|c|c|c|c|c|}
\hline Line. & Wave-length & Index. & $\theta$ from sin. & $\theta$ from arc. & $\theta$ from sin. & $\theta$ from arc. \\
\hline 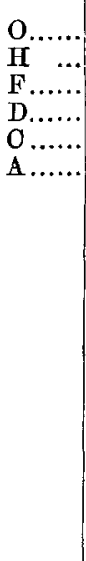 & $\begin{array}{l}0.34400 \\
0.39679 \\
0.48606 \\
0.58890 \\
0.65618 \\
0.76009 \\
0.815 \\
0.850 \\
0.890 \\
0.910 \\
0.940 \\
1 \cdot 130 \\
1.270 \\
1.360 \\
1.540 \\
1.580 \\
1.810 \\
1.870 \\
1.980 \\
2.030\end{array}$ & $\begin{array}{l}1.6266 \\
1.6070 \\
1.5899 \\
1.5798 \\
1.5757 \\
1.5714 \\
1.5697 \\
1.5687 \\
1.5678 \\
1.5674 \\
1.51668 \\
1.5636 \\
1.5616 \\
1.5604 \\
1.5576 \\
1.5572 \\
1.5544 \\
1.5535 \\
1.5520 \\
1.5515\end{array}$ & \begin{tabular}{rrr}
28 & 21 & \multicolumn{6}{c}{} \\
24 & 18 & 40 \\
19 & 38 & 10 \\
16 & 6 & 20 \\
14 & 24 & 57 \\
12 & 24 & 20 \\
11 & 33 & 46 \\
11 & 4 & 50 \\
10 & 34 & 30 \\
10 & 20 & 30 \\
10 & 0 & 21 \\
8 & 18 & 43 \\
7 & 23 & 19 \\
6 & 53 & 56 \\
6 & 9 & 30 \\
5 & 56 & 0 \\
5 & 10 & 40 \\
5 & 0 & 40 \\
4 & 43 & 55 \\
4 & 37 & 11
\end{tabular} & $\begin{array}{rrrr}28 & 2.1 & 50 \\
& \text { do. } \\
19 & 38 & \\
19 & 38 & 7 \\
16 & 6 & 26 \\
14 & 24 & 57 \\
12 & 24 & 20 \\
11 & 33 & 42 \\
11 & 4 & 43 \\
10 & 34 & 28 \\
10 & 20 & 23 \\
10 & 0 & 21 \\
8 & 18 & 23 \\
7 & 22 & 53 \\
6 & 53 & 15 \\
6 & 8 & 31 \\
5 & 54 & 20 \\
5 & 9 & 10 \\
4 & 59 & 10 \\
4 & 42 & 20 \\
4 & 35 & 17\end{array}$ & $\begin{array}{rrr}29 & 16 & 30 \\
25 & 5 & 0 \\
20 & 14 & 50 \\
& & \\
14 & 51 & 15\end{array}$ & $\begin{array}{lll}29 & 17 & 110 \\
20 & 15 & 33 \\
14 & 52 & 25\end{array}$ \\
\hline
\end{tabular}

Tarle II.-Rock-salt Prism.

(Prof. Langley's Determinations, Phil. Mag. May 1886 , no. 132.)

\begin{tabular}{|c|c|c|c|c|c|c|c|}
\hline & \multirow{2}{*}{$\begin{array}{l}\text { I. Wave- } \\
\text { length. }\end{array}$} & \multirow{2}{*}{ Index. } & \multirow{2}{*}{$\begin{array}{l}\theta \text { from } \\
\text { sine. }\end{array}$} & \multirow{2}{*}{$\begin{array}{l}\theta \text { from } \\
\text { arc. }\end{array}$} & \multirow{2}{*}{$\begin{array}{l}\text { Differ- } \\
\text { ence. }\end{array}$} & \multicolumn{2}{|c|}{ Limit of Refract. } \\
\hline & & & & & & Sine. & Arc. \\
\hline M & 0.3727 & 1.57486 & 24463 & 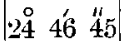 & $+\dot{0} 10$ & & \\
\hline & 03820 & 1.57207 & 2485 & $\begin{array}{lll}24 & 8 & 19\end{array}$ & 014 & & \\
\hline $\mathbf{H}_{2} \ldots$ & 0.3933 & 1.56920 & $23 \quad 2350$ & $\mid 23 \quad 24 \quad 8$ & 018 & & \\
\hline $\mathrm{H}_{1}^{2} \ldots$ & $0 \cdot 3968$ & $\mathbf{1} \cdot 56833$ & 231049 & datum. & & 1.52570 & \\
\hline G $\ldots$ & 0.4303 & 1.56133 & 21170 & $\begin{array}{lll}31 & 17 & 7\end{array}$ & & & \\
\hline F....... & $0 \cdot 4861$ & 155323 & 184447 & $\begin{array}{lll}18 & 44 & 47\end{array}$ & 00 & & \\
\hline & 0.5167 & 1.54991 & 173541 & 173540 & -0 & & \\
\hline & 0.5183 & 1.54975 & $1732 \quad 19$ & $\begin{array}{lll}17 & 32 & 16\end{array}$ & -03 & & \\
\hline $\mathrm{D}_{2} .$. & 0.5889 & $1 \% 4+18$ & 152249 & 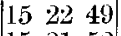 & 0 & & \\
\hline $\mathrm{D}_{3}^{2}$ & 0.5895 & 1.54414 & 152150 & $\begin{array}{lll}15 & 21 & 52\end{array}$ & +0 & & \\
\hline C... & 06562 & $1.5405 \mathrm{l}$ & $\begin{array}{lll}13 & 46 & 12\end{array}$ & $1346 \quad 12$ & 0 & & \\
\hline B.. & 0.6867 & 1.53919 & $12 \quad 849$ & 12850 & +0 & & \\
\hline A...... & 0.7601 & $1 \cdot 53670$ & $1151 \quad 12$ & $\begin{array}{lll}11 & 51 & 13\end{array}$ & +0 & & \\
\hline & $0 \cdot 94$ & $1 \cdot 5328$ & 93353 & 93358 & +0 & & \\
\hline & $1 \cdot 13$ & $1 \div 305$ & $756 \quad 42$ & $\begin{array}{llll}7 & 56 & 40\end{array}$ & -02 & & \\
\hline & $1 \cdot 38$ & 1.5287 & 62949 & $6 \quad 2951$ & +02 & & \\
\hline$\Omega \ldots \ldots$ & 1.82 & 1.5268 & 45524 & $\begin{array}{lll}4 & 55 & 14\end{array}$ & -010 & 1.5251 & $1 \cdot 5249$ \\
\hline
\end{tabular}

$H_{l}$ is found from indices of $H_{1}$ and $A$, and from $H$ all the other lines are deduced. Molecular distance $h=0.032570 ; \mu_{\mathrm{B}}-3\left(\mu_{F}-\mu_{B}\right)=1.52621$. 
TABLE III.

\begin{tabular}{|c|c|c|c|c|c|c|c|}
\hline \multicolumn{8}{|c|}{ Bisulphide of Carbon. } \\
\hline $\begin{array}{c}\text { Temp. } \\
t .\end{array}$ & $\underset{\substack{\text { Sp. Grav } \\
d .}}{\mid}$ & $\underset{\mu_{\Delta .}}{\text { Index } A}$ & $\begin{array}{c}\text { Index } \mathrm{H} \\
\mu_{\mathbf{x}} \cdot\end{array}$ & $\underset{\theta_{\mathrm{H}}}{\operatorname{Angle} \mathrm{A}}$ & $\begin{array}{c}\text { Limit. } \\
\nu .\end{array}$ & $\begin{array}{c}\text { Mol. dist. } \\
h .\end{array}$ & $\begin{array}{c}\text { Specific } \\
\text { Limit. } \\
\frac{v-1}{d}\end{array}$ \\
\hline $\begin{array}{r}1 \cdot 5 \\
230 \\
38 \cdot 0\end{array}$ & $\begin{array}{l}1 \cdot 2909 \\
1 \cdot 2594 \\
1 \cdot 2494\end{array}$ & $\begin{array}{l}1 \cdot 6227 \\
1 \cdot 6070 \\
1 \cdot 6026\end{array}$ & $\begin{array}{l}1.7159 \\
1.6972 \\
1.6924\end{array}$ & $\left|\begin{array}{rrr}18 & 38 & 2 \\
18 & 38 & 2 \\
18 & 25 & 50 \\
18 & 25 & 30\end{array}\right|$ & $\begin{array}{l}1 \cdot 5944 \\
1 \cdot 5799 \\
1 \cdot 5752\end{array}$ & $\begin{array}{l}\cdot 048505 \\
\cdot 04814 \\
\cdot 048559\end{array}$ & $\begin{array}{l}46048 \\
46046 \\
46038\end{array}$ \\
\hline \multicolumn{8}{|c|}{ Another Specimen. } \\
\hline $\begin{array}{l}10 \cdot 0 \\
24 \cdot 5\end{array}$ & $\begin{array}{l}1.2793 \\
1.2593\end{array}$ & $\begin{array}{l}1.6153 \\
1.6045\end{array}$ & $\left|\begin{array}{l}1.7078 \\
1.695 t\end{array}\right|$ & $\left|\begin{array}{lll}18 & 37 & 43 \\
18 & 30 & 45\end{array}\right|$ & $\begin{array}{l}1.5869 \\
1.5770\end{array}$ & $\mid \begin{array}{l}048673 \\
\cdot 048616\end{array}$ & $\begin{array}{l}45885 \\
45819\end{array}$ \\
\hline \multicolumn{8}{|c|}{$\begin{array}{l}\text { Comparison of Values of Limit calculated from sine } \theta_{\mathrm{H}} \text { and are } \theta_{\mathrm{B}} \text { with } \\
\text { corresponding angles for } \mathrm{CS}_{2} \text { at } 10^{\circ}-\theta_{\mathrm{g}}=37^{\circ} 44^{\prime} 40^{\prime \prime} \text {. }\end{array}$} \\
\hline & Line $\mathrm{A}$. & B. & C. & D. & E. & F. & G. \\
\hline $\begin{array}{l}\text { Sine } \ldots \\
\text { Limit... }\end{array}$ & $\mid \begin{array}{cc}18 & 374 \\
1.5870\end{array}$ & $\mid \begin{array}{ccc}0 & 4 & 4 \\
2 & 50 & 0\end{array}$ & (..... & $\left|\begin{array}{ccc}0 & 19 & 19 \\
1 & 15862 & 59\end{array}\right|$ & …... & $\begin{array}{l}2954 " 4 \\
1.5843\end{array}$ & $\begin{array}{l}341940 \\
1.5847\end{array}$ \\
\hline $\begin{array}{l}\text { Arc ... } \\
\text { Limit... }\end{array}$ & $\left|\begin{array}{ccc}18 & 39 & 46 \\
1 & 5869\end{array}\right|$ & $\left|\begin{array}{ccc}20 & 41 & 52 \\
\ldots \ldots\end{array}\right|$ & $\begin{array}{l}\ldots \ldots . \\
\ldots \ldots .\end{array}$ & $\left|\begin{array}{ccc}24 & 18 & 52 \\
1 & 5861\end{array}\right|$ & $\begin{array}{l}\ldots . . . \\
\ldots \ldots .\end{array}$ & \multicolumn{2}{|c|}{ 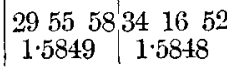 } \\
\hline $\begin{array}{l} \\
\\
\text { Sine } \quad \ldots \\
\text { Arc } \quad \cdots\end{array}$ & $\begin{array}{l}\text { Another va } \\
\left|\begin{array}{ccc}8 & 4 & 4 \\
18 & 35 & 20 \\
18 & 35 & 10\end{array}\right| \\
\text { The }\end{array}$ & $\begin{array}{l}\mid \begin{array}{ccc}0 & \\
20 & 40 & 10 \\
20 & 40 & 40\end{array} \\
\text { e value of }\end{array}$ & 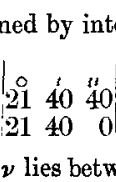 & $\begin{array}{l}\text { terpolation, } \\
\left|\begin{array}{lll}\circ & 1 \\
24 & 16 & 50 \\
24 & 15 & 30\end{array}\right| \\
\text { ween } \\
\text { ween }\end{array}$ & 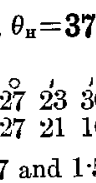 & $\begin{array}{l}29^{\prime} 20^{\prime \prime} \\
295450 \\
295150 \\
852 .\end{array}$ & $\mid \begin{array}{lll}0 & 1 \\
34 & 13 & 40 \\
34 & 11 & 10\end{array}$ \\
\hline \multicolumn{8}{|c|}{ Mint $\mathrm{Hydrocarbon.}$} \\
\hline $\begin{array}{l}\text { Temp. } \\
t .\end{array}$ & $\mid \begin{array}{c}\text { Sp. Grav. } \\
d .\end{array}$ & $\mid \begin{array}{c}\text { Index A. } \\
\mu_{\mathrm{A}}\end{array}$ & $\underset{\mu_{\mathrm{r}}}{\text { Index } \mathrm{H}}$ & - $\underset{\theta_{A}}{\text { Angle } A .}$ & $\begin{array}{l}\text { Limit. } \\
\nu .\end{array}$ & $\underset{h .}{\text { Mol. dist. }}$ & $\begin{array}{l}\text { Specific } \\
\text { Limit. } \\
\frac{\nu-1}{d}\end{array}$ \\
\hline $\begin{array}{r}6 \circ 5 \\
25 \cdot 0\end{array}$ & $\begin{array}{l}0.8926 \\
0.8820\end{array}$ & $\begin{array}{l}1.4728 \\
1.4650\end{array}$ & $\begin{array}{l}1.4988 \\
1.4901\end{array}$ & $\left|\begin{array}{rrr}11 & 4 & 50 \\
10 & 58 & 0\end{array}\right|$ & $\begin{array}{l}1 \cdot 4632 \\
1 \cdot 4561\end{array}$ & $\begin{array}{l}\ldots \ldots . \\
\ldots . . .\end{array}$ & $\begin{array}{l}51893 \\
51712\end{array}$ \\
\hline \multicolumn{8}{|c|}{ Benzene. } \\
\hline $\begin{array}{r}2 \cdot 0 \\
23 \cdot 7 \\
28 \cdot 6\end{array}$ & $\begin{array}{l}0.8979 \\
0.8760 \\
0.8709\end{array}$ & $\begin{array}{l}1 \cdot 5021 \\
1 \cdot 4893 \\
1 \cdot 4860\end{array}$ & $\begin{array}{l}1 \cdot 5460 \\
1 \cdot 5320 \\
1 \cdot 5279\end{array}$ & 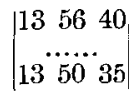 & \begin{tabular}{|l|l}
$1 \cdot 4873$ \\
$1 \cdot 4725$ \\
$1 \cdot 4720$
\end{tabular} & $\begin{array}{r}039219 \\
039122 \\
.038892\end{array}$ & $\begin{array}{l}54272 \\
5+328 \\
54196\end{array}$ \\
\hline
\end{tabular}


Table III. (continued).

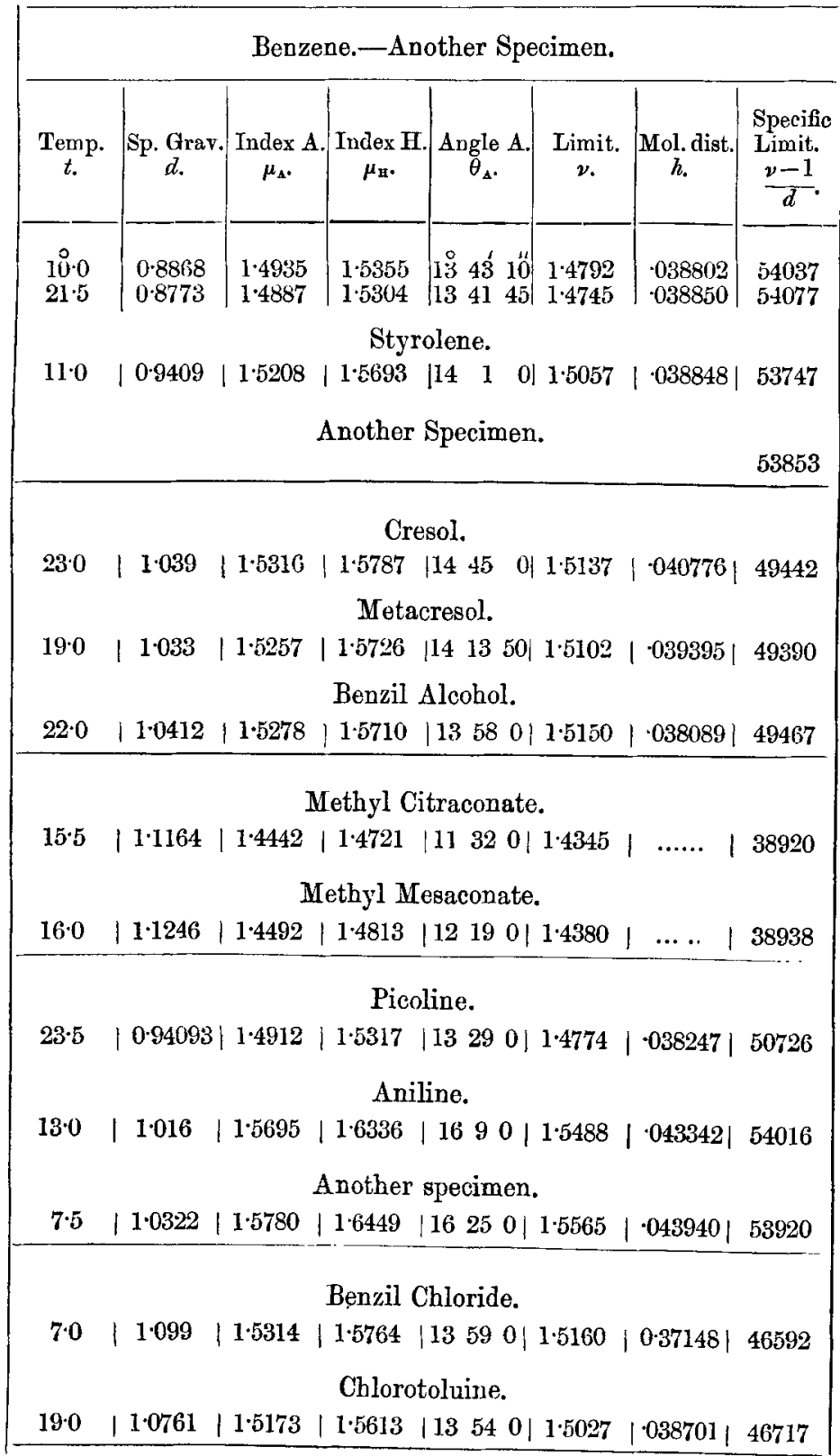


the Wave-length within a Refractive Medium.

Table III. (continued).

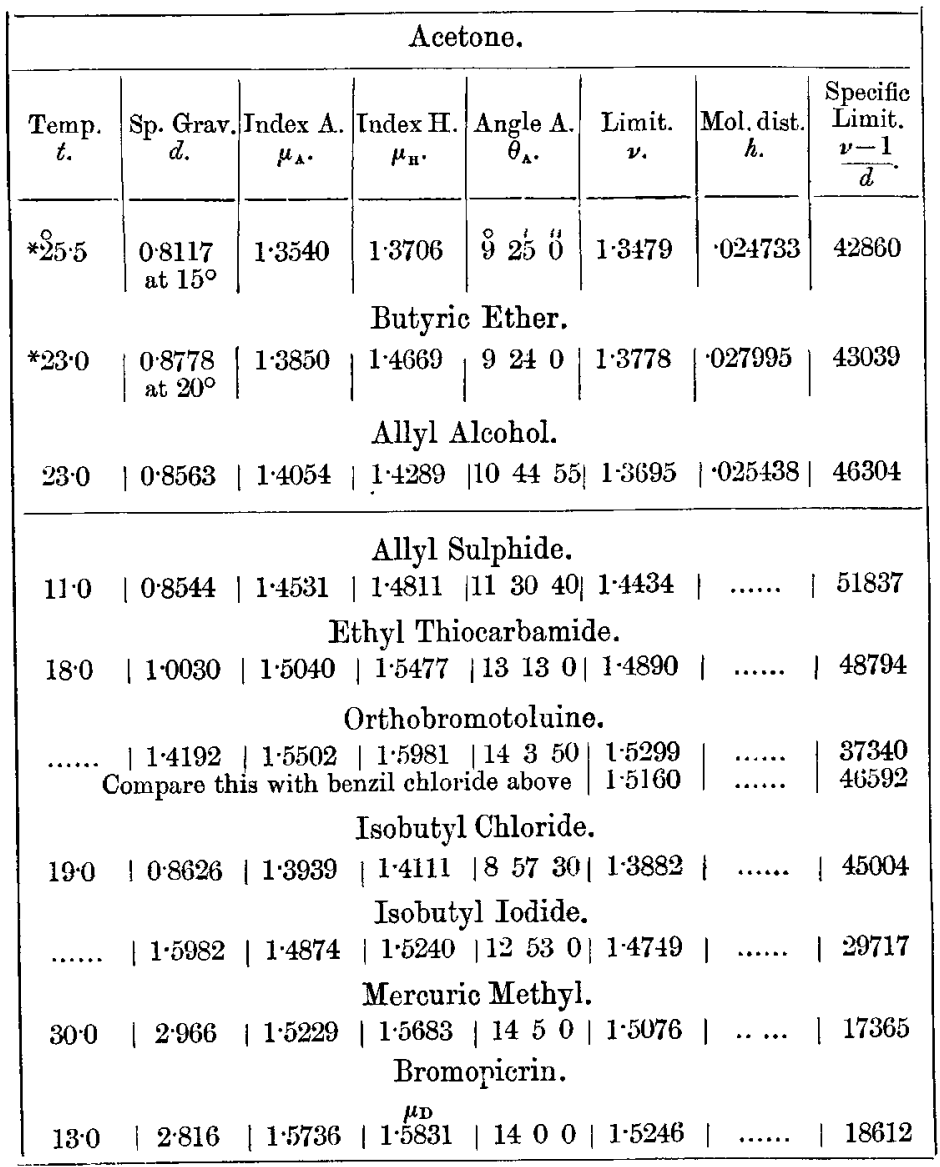

* It will be seen that in both these $d$ is too great.

TABLE IV.

\begin{tabular}{|c|c|c|c|c|c|}
\hline Substance. & $\begin{array}{l}\text { Lower } \\
\text { Index. }\end{array}$ & $\begin{array}{l}\text { Upper } \\
\text { Index. }\end{array}$ & Angle $\theta_{\mathrm{A}}$. & Limit. & $\begin{array}{c}\text { Limiting } \\
\text { Wave-length }\end{array}$ \\
\hline Selenium & $\begin{array}{r}\mu_{\mathrm{A}} \\
2653\end{array}$ & $\underset{2980}{\mu_{\mathrm{b}}}$ & $44^{\circ} \stackrel{\theta_{\Lambda .}}{9^{\prime}} 0^{\prime \prime}$ & $\begin{array}{c}\boldsymbol{\nu} \cdot \\
2 \cdot 1353\end{array}$ & 52957 \\
\hline $\begin{array}{c}\text { Solid } \\
\text { Sulphur }\end{array}$ & $\begin{array}{c}\mu_{\mathrm{A} \cdot} \\
1^{\circ} \cdot 9024\end{array}$ & $\begin{array}{c}\mu_{\mathrm{E}} \\
1.9527\end{array}$ & $20^{\circ} 30^{\prime} 0^{\prime \prime}$ & $1 \cdot 8621$ & $2663 \cdot 8$ \\
\hline $\begin{array}{c}\text { Solid } \\
\text { Phosphorus }\end{array}$ & $\begin{array}{c}\mu_{\mathrm{A} .} \cdot 1059 \\
2 \cdot 1\end{array}$ & $\begin{array}{l}\mu_{E} \\
2 \cdot 1442\end{array}$ & $20^{\circ} 40^{\prime} 0^{n}$ & $2 \cdot 0611$ & $2683 \cdot 8$ \\
\hline
\end{tabular}


TABLE $V$.

\begin{tabular}{|c|c|c|c|c|}
\hline Sp. g. & $\mu_{\mathbf{\Delta}}$ & $\mu_{\mathbf{H} .}$ & $\nu$. & $\frac{\nu-1}{d}$. \\
\hline $\begin{array}{c}\text { Water. } \\
1.000\end{array}$ & 1.32924 & 1.34393 & 1.3249 & 3249 \\
$\begin{array}{c}\text { Alcohol. } \\
07919\end{array}$ & 1.3585 & 1.3735 & 1.3531 & 44589 \\
\hline
\end{tabular}

\section{Tidal Action as an Agent of Geological Change. By T. Mellard Reade, C.E., F.G.S., F.R.I.B.A.*}

THE nature of tidal action, as regards its effect on the sea1 bottom, seems to be insufficiently understood, if not in many cases actually misapprehended.

In looking over most of the late works on Geology this fact has been much impressed upon me. Very little influence seems to be attached to the tides as agents of geological change, and then only as surface-currents affecting the coasts and shores. So long ago as 1873 , in a paper entitled "Tidal Action as a Geological Cause" $\uparrow$, I attempted to show that the mechanical action of the tides was deep and wide spread, affecting not only the littoral, but the whole bottom of the sea, while the profoundest depths of the ocean are not entirely exempt from tidal movement.

My present object, while recalling attention to this subject, is to adduce some further facts in support of the important office the tides perform as distributers of material worn from the land, and even in special cases as agents of erosion acting on the sea-bottom.

The force producing the tidal-wave affects every particle equally, down to the most profound depths of the ocean, while the forces creating wind-waves act only on the surface. A cork floating upon deep water disturbed only by the ordinary waves, revolves (as do also all the surface-particles of water) either in a vertical circle or an ellipse, not very different from one having the longer axis vertical. In the free tide-wave

* Communicated by the Author.

$\dagger$ Proceedings of the Geological Society of Liverpool, Session 1873-4. 